\title{
A Multi-Objective Optimization Method to inte- grate Heat Pumps in Industrial Processes
}

\author{
Helen Becker, Giulia Spinato, François Maréchal* \\ Industrial Energy Systems Laboratory (LENI), Ecole Polytechnique Fédérale de Lau- \\ sanne, CH-1015 Lausanne, Switzerland
}

\begin{abstract}
Aim of process integration methods is to increase the efficiency of industrial processes by using pinch analysis combined with process design methods. In this context, appropriate integrated utilities offer promising opportunities to reduce energy consumption, operating costs and pollutants emissions. Energy integration methods are able to integrate any type of predefined utility, but so far there is no systematic approach to generate potential utilities models based on their technology limits. This work focusses on the integration of industrial heat pumps and the development of a corresponding heat pump data base. This latter offers the possibility to integrate different heat pump types to any process, in a flexible and systematic way. A methodology, integrating the heat pump data base in an energy integration problem, and using multi objective optimization in order to identify optimal solutions, is presented. The results of a brewery process are presented and analyzed.
\end{abstract}

Keywords: energy integration, pinch analysis method, utility integration, industrial heat pumps, process design, multi-objective optimization

\section{Introduction}

Pinch analysis is a powerful tool allowing the minimization of the energy consumption of a process, both in terms of hot utility (often related to fuel combustion) and cold utility (often subject to environmental constraints). In this context, heat pump integration plays an important role in the rational use of energy conversion. A correctly placed heat pump upgrades part of the heat available below the pinch point (heat source), making it available above the pinch point (heat sink). Therefore, both heating and cooling requirements are reduced. In practice, an appropriate integration of heat pumps requires the identification of the optimal heat pump(s) and of its operating conditions. In the literature, different approaches for process integration can be found: Wallin and Berntsson (1990) propose a methodology to optimize the temperatures of the heat source and the heat sink, the size of the heat pumps and the choice of the streams used by the heat pump. Bagajewicz and Barbaro (2003) consider temperature levels as decision variables in order to avoid discrete temperature levels which need a fine interval partition to find good solutions. This approach could lead to non-realistic solutions, due to the fact that generally, industrial heat pumps work with one condenser and one evaporator. In Becker et al. (2011), a methodology to estimate the potential of heat pump integration based on pinch analysis is reported. In the frame of an optimization process, it considers heat recovery between process streams together with the integration of energy conversion systems. However the integrated utilities (including heat pumps) are chosen from a predefined and limited list.

*helen.becker@epfl.ch 
The following work represents the next step, that consists in a systematic methodology in which practical constraints of the technology (e.g. volumetric flow-rate operating ranges for different compressor technology or temperature ranges for given refrigerant) are considered at the targeting stage. A structured heat pump data base has been developed in order to provide realistic heat pump models. In this way, the final heat pump solutions of this generic approach are not only optimal but also realistic.

\section{Method}

The multi-objective optimization strategy chosen in this work is based on the decomposition of the optimization problem in master and slave sub-problems (Fig. 1) (Gassner and Maréchal, 2009). The two objectives are minimizing the operating costs of the industrial process and the investment costs related to new heat pump installations. At the master level, the values of the decision variables are chosen by the evolutionary algorithm. At this step, the operating conditions of the heat pumps (condensation and evaporation temperatures) are fixed, enabling the thermodynamic model to calculate the heat pump cycles and the enthalpy-temperature profiles of heat pumps hot and cold streams. Using

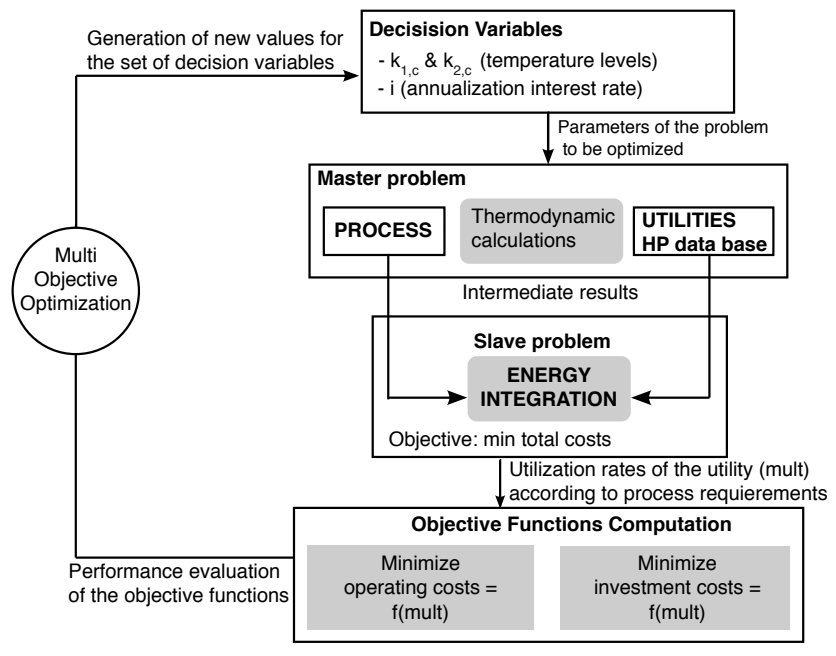

Figure 1: Optimization problem

the operating conditions of the heat pumping cycles, the slave problem solves the energy integration problem, which aims at minimizing the total cost, including the yearly operating costs of hot and cold utilities and the annualized investment costs of heat pump installations. This model optimizes all utility flow rates simultaneously, using the mixed integer linear programming (MILP) formulation of the heat cascade. Finally, the corresponding operating and investment costs can be computed. The master problem is solved using an evolutionary algorithm. This allows using complex heuristics in the heat pump selection procedure and process integration, without difficulties related to constraints and non-differentiable equations. The presented optimization approach results in a set of optimal solutions laying on a Pareto front. Considering that the goal is to help the engineers in their final decision, this method gives the advantage that the final solution can be chosen among the optimal solutions by applying financial, environmental or other criteria. 


\section{Definition of the heat pump data base}

The heat pump technologies are modeled for 8 compressor technologies in terms of operating conditions ranges (volumetric flow-rate, pressure ratio, temperature levels) and isentropic efficiency. To be able to integrate several heat pumps using the same compressor technology and multi-stage heat pumps, each compressor technology has been implemented 4 times. Hence, the heat pump data base consists in $8 * 4$ closed compression cycles per refrigerant. A cycle group $(c g)$ is composed of 8 cycles with different compressors (in total 4 cycle groups). The data base has been developed in such a way that any new heat pump cycle or type can be easily added.

\section{Optimization algorithm}

The multi-objective optimization approach aims at minimizing operating costs and investment costs whereas the slave problem optimizes the total costs, defined by the sum of the yearly operating costs and the annualized investment costs (Eq. (1)). The annualized investment is calculated as a function of the interest (the decision variable $i$ ) and a live time of 20 years. The investment rate $i[0,20]$ is added to the set of decision variables in order to influence the weight of investment costs when calculating the total costs (TC).

$$
F_{\text {obj,slave }}=T C=O p C+\operatorname{Inv} C\left(\frac{i(i+1)^{n}}{(i+1)^{n}-1}\right)
$$

For each cycle group $\left(c_{g}\right)$, two supplementary decision variables are necessary $\left(k_{1, c g}\right.$, $\left.k_{2, c g}\right)$. The parameters $k_{1, c g}[0.1,0.9]$ and $k_{2, c g}[0.1,1]$ define the evaporation $\left(T_{\text {eva,cg }}\right)$ and condensation $\left(T_{\text {cond }, c g}\right)$ levels of the heat pumps as a function of the operating range of the refrigerant $\mathrm{f}\left(T_{\text {cond,max }}(f)\right.$ and $\left.T_{\text {eva,min }}(f)\right)$ as defined in Eqs. (2), (3) and in Fig. 2.

$$
\begin{aligned}
& T_{\text {cond }, c}=T_{\text {cond,max }}(f)-k_{1, c} \cdot\left(T_{\text {cond }, \max }(f)-T_{\text {eva,min }}(f)\right) \\
& T_{\text {eva }, c}=T_{\text {eva,min }}(f)+k_{2, c} \cdot\left(T_{\text {cond }, c}-T_{\text {eva,min }}(f)\right)
\end{aligned}
$$

As reported in Fig. 1, the values of the decision variables are sent to the master level problem where the thermodynamics of the model are solved. Then a MILP problem, that

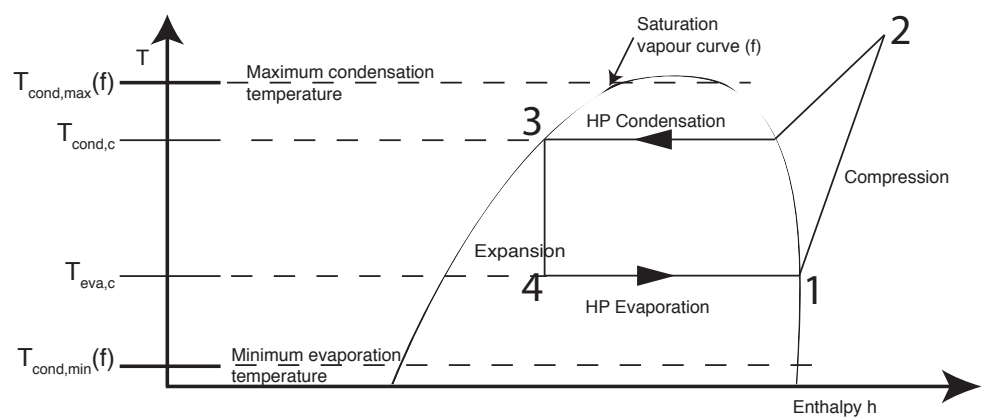

Figure 2: Heat pump temperature levels

minimizes the total costs (Eq. (1)), is solved. The complete formulation can be found in Becker et al. (2011). The yearly operating costs (OpC) are defined in Eq. (4), whereas 
the investment costs (InvC) are estimated with Eq. (5) according to available correlations, as a function of the compressor power. The optimal utilization rates of the heat pumps are calculated by the energy integration model, together with the process units and other utilities present in the process. In particular, multi-stage heat pumps, composed by several single-stage cycles, could be integrated in the same manner.

$$
O p C=c_{f u e l} \dot{E}_{f u e l}^{+}+c_{e l}^{+} \dot{E}_{e l}^{+}-c_{e l}^{-} \dot{E}_{e l}^{-}
$$

In Eq. (4) $\dot{E}_{\text {fuel }}^{+}$is the energy provided by the fuel (i.e. natural gas) purchased at the price $c_{f u e l}, \dot{E}_{e l}$ is the electrical power purchased $\left(^{+}\right)$or sent to the grid $\left(^{-}\right)$. The same notation is used to define the cost of purchased and sold electricity $c_{e l}$.

$$
\operatorname{Inv} C=\sum a \cdot\left(\dot{E}_{e l_{H P_{c}}}^{+}\right)^{b}
$$

The effective requirements of the process are known and it is possible to compute the (yearly) operating costs [kEuro/year] and the investment costs [kEuro]. Then, the optimizer evaluates them and generates new values for the decision variables able to minimize both objective functions. After a certain number of iterations the optimal solutions could be plotted on a Pareto curve.

\section{Example of application}

Brewery process transforms water and malt into beer after several stages of heating and cooling of the product, whose operating conditions are fixed by process practice. Refrigeration and auxiliary systems, such as cleaning in place, are present. More details could be found in Dumbliauskaite et al. (2010). The data base in this example uses two refrigerants (R717 and R134a). Using the cited case study and the approach presented in this paper, the Pareto front in Fig. 3a is obtained after 1000 iterations. The energy flows and

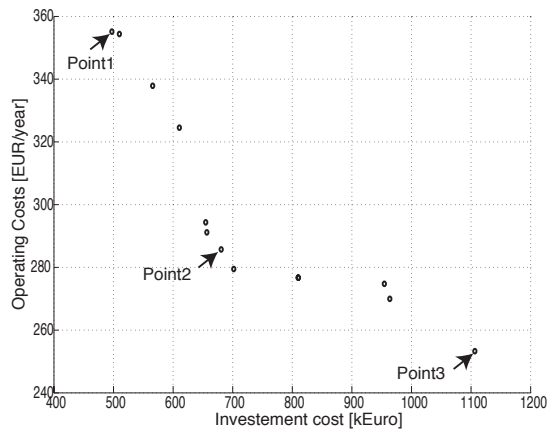

(a) Pareto front

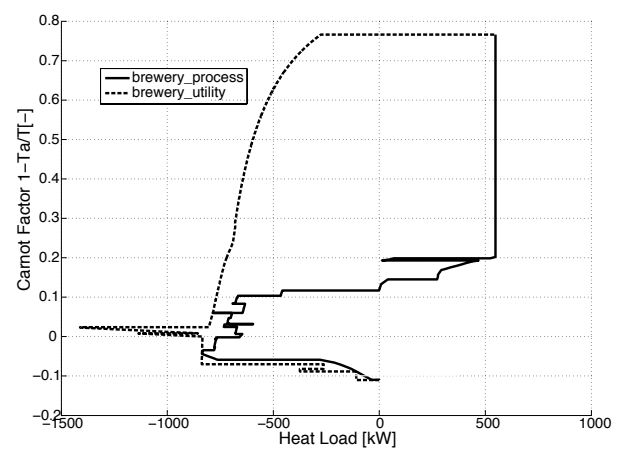

(b) Integrated composite curves of utilities of point 1

Figure 3: Optimization results

the operating and investment costs of three interesting points are shown in Tab. 1: Point1 corresponds to the minimum investment costs and consists in 3 heat pump cycles used for refrigeration (Fig. 3b), as required to satisfy the cooling demand of the process. In point 2 two supplementary heat pumps are integrated (Fig. 4a). This latter upgrades heat, reducing consequently the operating costs but small supplementary investment costs are necessary. Finally, Point 3 corresponds to the minimum operating costs (Fig. 4b). 


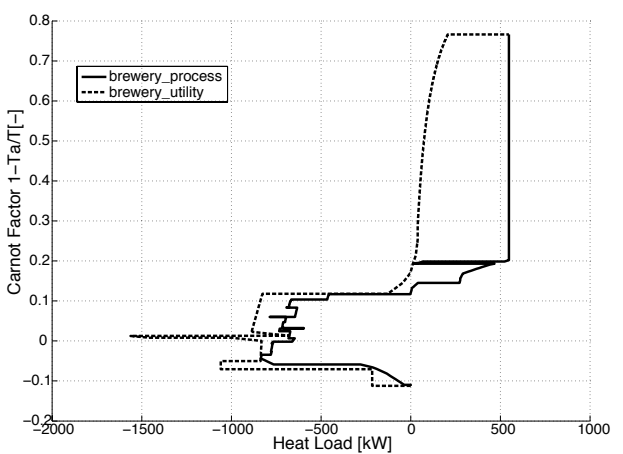

(a) Integrated composite curves of utilities of point2

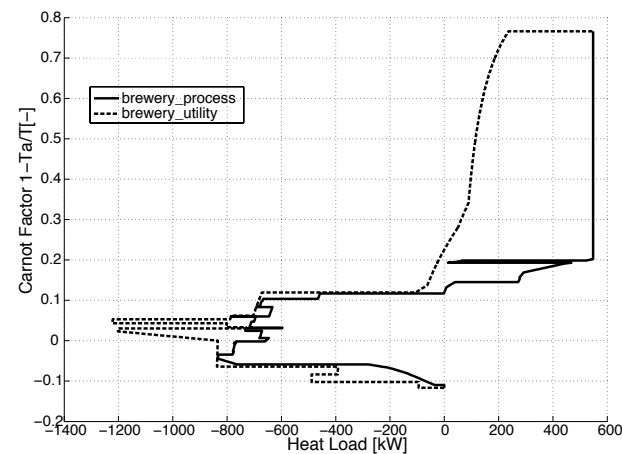

(b) Integrated composite curves of utilities of point 3

Figure 4: Integrated composite curves of utilities

Table 1: Results

\begin{tabular}{|l|r|r|r|r|r|r|}
\hline & $\begin{array}{r}\text { InvC } \\
{[\mathrm{kEuro}]}\end{array}$ & $\begin{array}{r}\text { OpC } \\
{[\mathrm{kEuro} / \mathrm{year}]}\end{array}$ & $\begin{array}{r}\text { Fuel } \\
{[\mathrm{kW}]}\end{array}$ & $\begin{array}{r}\text { Cooling water } \\
{[\mathrm{kW}]}\end{array}$ & $\begin{array}{r}\text { Electricity } \\
{[\mathrm{kW}]}\end{array}$ & $\begin{array}{r}\text { HP units } \\
{[-]}\end{array}$ \\
\hline point1 & 497.3 & 355.2 & 1485 & 860 & 206 & 3 \\
\hline point2 & 680.8 & 285.7 & 615.3 & 482.4 & 532.3 & 5 \\
\hline point3 & 1106.0 & 253.3 & 562.3 & 368.2 & 461.3 & 5 \\
\hline
\end{tabular}

\section{Conclusions and perspectives}

The approach used in the implementation of the heat pump data base allows the optimal integration of single stage, multistage heat pumps and even combination of cycles (i.e. for refrigeration). The data base approach allows to use specific models for each type of heat pump. They are integrated into the process using process integration models. It is implemented in such a way that any compressor technology or heat pump cycle configurations working with pure or mixture of refrigerants could be integrated. Moreover all the points on the Pareto curve represent optimal and practical feasible solutions to improve the process efficiency and that will be used to decide the final "best" solution.

\section{References}

Bagajewicz, M., Barbaro, A., 2003. On the use of heat pumps in total site heat integration. Computers and Chemical Engineering 27, 1707-1719.

Becker, H., Maréchal, F., Vuillermoz, A., 2011. Process integration and opportunity for heat pumps in industrial processes. International Journal of Thermodynamics, ECOS 2009 special issue, accepted.

Dumbliauskaite, M., Becker, H., Maréchal, F., 2010. Utility optimization in a brewery process based on energy integration methodology. Proceedings of ECOS 2010, 91-98.

Gassner, M., Maréchal, F., 2009. Methodology for the optimal thermo-economic, multi-objective design of thermochemical fuel production from biomass. Computers and Chemical Engineering 33 (3), 769-781.

Wallin, E. Franck, P., Berntsson, T., 1990. Heat pumps in industrial processes - an optimization methodology. Heat Recovery Systems \& CHP 10 (4), 437-446. 